\title{
NETWORK REPRESENTATION OF MULTI-CELL ACCELERATING STRUCTURES
}

\author{
J.-Y. Raguin \\ CERN, Geneva, Switzerland
}

\begin{abstract}
The analysis of the electrodynamic properties of a complete multi-cell accelerating structure using electromagnetic numerical simulation codes is presently at the edge of existing computer capabilities. To overcome this limitation, a network representation is proposed which derives the overall scattering transfer matrix of such multi-cell structures from single-cell data calculated using the commercial finite-element code HFSS. For a constant-impedance structure, computation of the eigenvalues of this matrix allows dispersion diagrams to be obtained. In the more general case, this formalism leads to a representation of the coupled-chain of cavities as a set of cascaded non identical multipoles.
\end{abstract}

\section{INTRODUCTION}

Several high-energy physics laboratories around the world are engaged in the design of high-luminosity $\mathrm{e}^{+} \mathrm{e}^{-}$ linear colliders aiming at a center-of-mass energy equal to or higher than $0.5 \mathrm{TeV}$. Most of the designs use highgradient, normal-conducting accelerating structures with varying degrees of damping and detuning to reduce the unwanted effects of transverse wakefields.

An essential step in the design of these accelerating structures is the characterization of their electrodynamic properties. Since the advent of modern computers, a plethora of codes has been written which allow a fast determination of such parameters as excitation frequency, modal field pattern, shunt impedance, quality factor as well as the modal dispersion characteristics. For instance, to determine the dispersive properties of periodic structures, a very popular method consists of computing the eigenfrequencies of a cavity composed of a set of identical cells terminated at both ends by open-circuit or short-circuit boundary conditions with electromagnetic codes such as SUPERFISH [1], URMEL [2], etc. The accuracy of the results obtained with these grid-oriented or finite-element codes, based on a spatial discretisation of the cavity, are however tributary to the dimensions of the problem to be analysed and to the number of mesh points used.

Another approach is to use the mode-matching technique or the method of moments. These techniques are based on an analytical representation of the fields in subregions of the accelerating structure where solutions of Maxwell's equations are given as an expansion of orthogonal and complete basis functions [3], [4], [5]. Imposing a continuity condition of the transverse electric and magnetic fields on the common discontinuity surfaces results in a set of equations from which the associated scattering matrices are deduced. The electrodynamic properties of the structure are then obtained by cascading the matrices associated with each discontinuity and the smooth-walled sections of waveguide. Although these techniques are very powerful since the properties of a whole accelerating structure can in principle be computed, they are restricted to structures of simple geometry.

Electromagnetic codes such as the finite-element code HFSS [7], which can compute the generalized scattering matrix of a cavity with a complex geometry and welldefined ports over a given frequency range, have been available for several years. This paper shows that a judicious use of these codes combined with a suitable post-processing enables some of the fundamental characteristics of accelerating structures to be quickly calculated. The power of this scattering matrix formulation is illustrated by using HFSS to obtain the modal dispersion curves of prototype accelerating structures that have been built and tested at CERN in the framework of the CLIC studies [6].

\section{THEORETICAL CONSIDERATIONS}

A cell of a conventional accelerating structure is modelled by a two-port network where each port may carry $N$ modes. The amplitude coefficients of the outgoing $N$ modal waves at each port are related to the amplitudes of the incoming waves by the overall scattering matrix:

$$
\left(\begin{array}{l}
\underline{b}^{(1)} \\
\underline{b}^{(2)}
\end{array}\right)=\left(\begin{array}{ll}
\underline{S}_{11} & \underline{S}_{12} \\
\underline{S}_{21} & \underline{S}_{22}
\end{array}\right)\left(\begin{array}{l}
\underline{a}^{(1)} \\
\underline{a}^{(2)}
\end{array}\right) .
$$

$\underline{a}^{(1)}, \underline{a}^{(2)}, \underline{b}^{(1)}$ and $\underline{b}^{(2)}$ are column vectors associated with the amplitudes of the $N$ modes while $\underline{S}_{i j}$ are $N \times N$ matrices.

The scattering transfer matrix formulation lends itself naturally to the characterization of an accelerating structure when considered as a coupled-chain of cells and modelled by cascaded networks:

$$
\left(\begin{array}{l}
\underline{b}^{(1)} \\
\underline{a}^{(1)}
\end{array}\right)=\left(\begin{array}{ll}
\underline{T}_{11} & \underline{T}_{12} \\
\underline{T}_{21} & \underline{T}_{22}
\end{array}\right)\left(\begin{array}{l}
\underline{a}^{(2)} \\
\underline{b}^{(2)}
\end{array}\right),
$$

where the submatrices $\underline{T}_{i j}$ are computed from the $\underline{S}_{i j}$ with 
the following relations:

$$
\begin{aligned}
& \underline{T}_{11}=\underline{S}_{12}-\underline{S}_{11}\left(\underline{S}_{21}\right)^{-1} \underline{S}_{22} \\
& \underline{T}_{12}=\underline{S}_{11}\left(\underline{S}_{21}\right)^{-1}, \\
& \underline{T}_{21}=-\left(\underline{S}_{21}\right)^{-1} \underline{S}_{22}, \\
& \underline{T}_{22}=\left(\underline{S}_{21}\right)^{-1} .
\end{aligned}
$$

The dispersive properties of accelerating structures of the constant-impedance type can be calculated from the transfer matrix formulation and the application of Floquet's theorem. For an infinitely long periodic structure, Floquet's condition imposes the following relations between the amplitudes of the outgoing modal waves at the second port and the amplitudes of the incoming modal waves at the first port:

$$
\underline{b}^{(2)}=\exp (-\gamma d) \underline{a}^{(1)}
$$

where $d$ is the period of the structure and $\gamma=\alpha+j \beta$ is the complex propagation constant of the structure eigenmode. Similarly, the amplitudes of the outgoing waves at the first port are related to the amplitudes of the incoming waves at the second port:

$$
\underline{b}^{(1)}=\exp (\gamma d) \underline{a}^{(2)}
$$

The combination of these relations with the definition of the transfer matrix leads to the following system of equations:

$$
\left[\left(\begin{array}{ll}
\underline{T}_{11} & \underline{T}_{12} \\
\underline{T}_{21} & \underline{T}_{22}
\end{array}\right)-\lambda\left(\begin{array}{cc}
\underline{I} & 0 \\
0 & \underline{I}
\end{array}\right)\right]\left(\begin{array}{l}
\underline{a}^{(2)} \\
\underline{b}^{(2)}
\end{array}\right)=0
$$

where the scalar variable $\lambda=\exp (\gamma d)$ and where $\underline{I}$ is the unit matrix. This system obviously constitutes an eigenvalue problem to be solved for the amplitudes and for the propagation constants of the periodic accelerating structure modes.

One of the approaches under investigation to damp the higher order modes and to reduce the transverse wakefields in the CLIC accelerating structure consists of coupling each cell to a set of four identical radial waveguides. These waveguides are dimensioned in such a manner that their lowest cutoff frequency is between the upper frequency of the fundamental passband and the lower frequency of the first dipole band [8]. Such an arrangement can also be studied using the above formulation, albeit with the following minor modifications. Taking into account the symmetry properties of the cell, it can be modelled by a three-port network, the third port being associated with a waveguide. The computation of the scattering matrix of this network and the assumption that the waveguide is terminated by its characteristic impedance lead to the same type of eigenvalue problem as above: the scattering submatrices related to the third port need not be considered. Such a technique is particularly useful for studying the dispersive properties of heavily loaded structures under ideal conditions.

\section{DISPERSIVE PROPERTIES OF CLIC ACCELERATING STRUCTURES}

Two structure geometries have been analysed to demonstrate the use of this technique. The technique was first tested on one of the first prototype CLIC accelerating structures built initially to work in the single-bunch regime. It consists of a constant-impedance cylindrically-symetric disk-loaded structure designed to operate at $29.985 \mathrm{GHz}$ in the $2 \pi / 3$ mode. The cell geometry and dimensions are given in [9]. Each port of the computational volume used in HFSS was set in the middle of a cell, halfway between two consecutive irises. The calculation of the associated eigenvalue problem by using standard techniques followed the computation of the scattering matrix. The dispersion characteristics covering the fundamental passband are shown in Figure 1 for half of the first Brillouin zone.

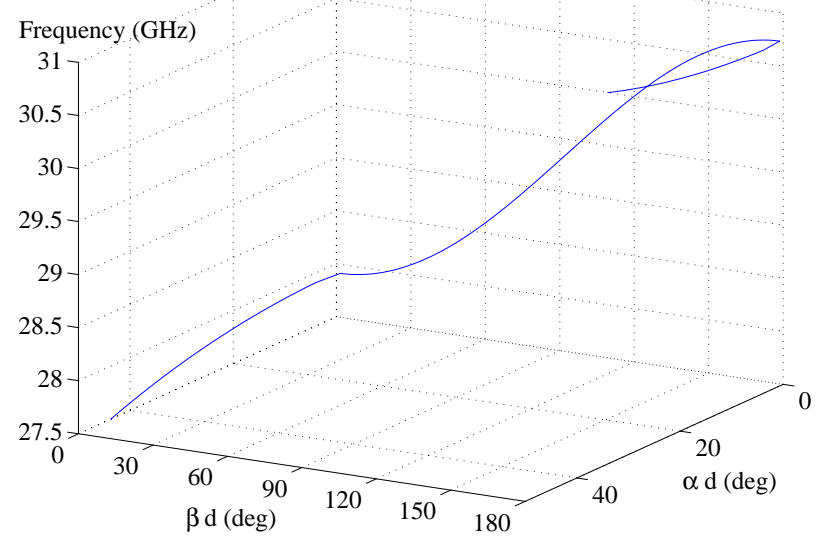

Figure 1: Dispersion diagram associated with the fundamental passband for the single-bunch CLIC accelerating structure

In this case, it has been assumed that the structure is lossless and that only one mode, a $\mathrm{TM}_{01}$ waveguide mode, is excited at each port.

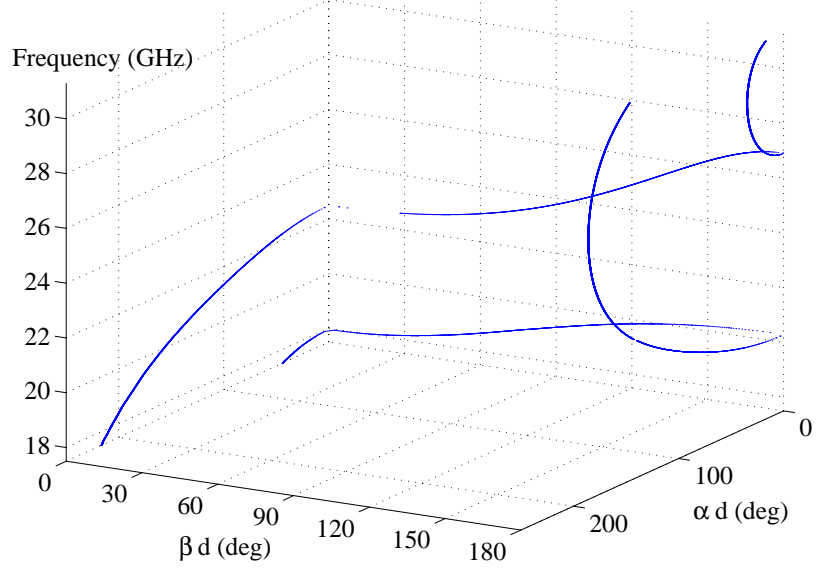

Figure 2: Dispersion diagram for the first two dipole passbands of a modified cell of the TDS without coupled waveguides 
Note that, since no propagation direction has been imposed, both $+\gamma$ and $-\gamma$ are true propagation constants for the eigenmodes of the structure. In addition, both $+\gamma+$ $j 2 \pi n / d$ and $-\gamma+j 2 \pi n / d$, where $n$ is an integer, are also solutions due to the multivalued nature of the logarithm function.

The second example concerns a tapered damped accelerating structure (TDS) [10]. Figure 2 shows the dispersion diagram associated with the first two dipole passbands when no waveguide is coupled to the cell. In the presence of damping with coupled waveguides, the dispersion properties are modified in such a manner that complex modes (modes with both $\alpha$, the attenuation constant, and $\beta$ not equal to zero) appears. For moderately large coupling holes, these types of mode are predominant in the band edge regions (Figure 3 ).

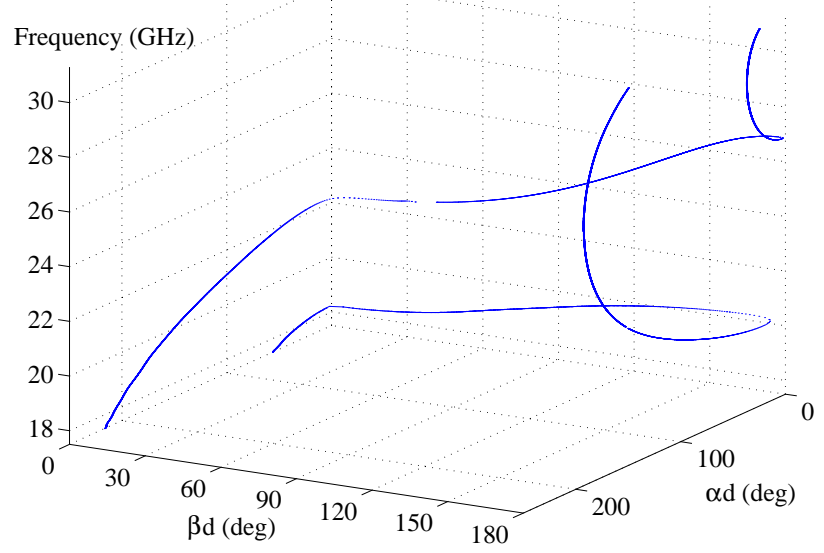

Figure 3: Dispersion diagram for the first two dipole passbands of a modified cell of the TDS with moderate waveguide coupling

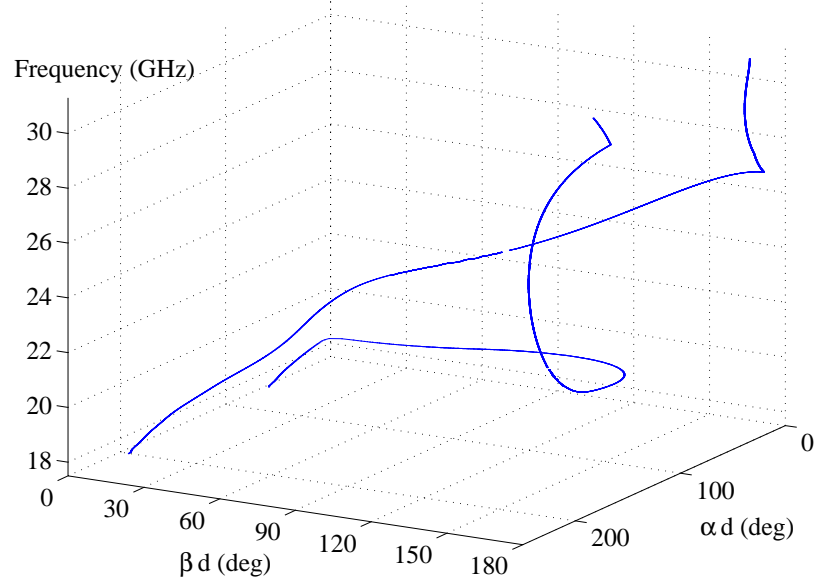

Figure 4: Dispersion diagram for the first two dipole passbands of a modified cell of the TDS with strong waveguide coupling

For a strong coupling (Figure 4), the dispersive properties are clearly very different from the case without coupling waveguides: attenuation is shown to take appreciable val- ues within the whole frequency range shown.

\section{CONCLUSIONS}

The use of electromagnetic codes to compute the scattering matrix of a unit cell of a constant-impedance accelerating structure, modelled as a two-port network, each port carrying a particular number of modes, combined with appropriate post-processing, can provide modal dispersion characteristics. The robustness of the technique has been demonstrated on the CLIC single-bunch structure and on a cell of a tapered damped structure. For a non-periodic structure, the networks associated with each cell, including those related to the input and output couplers, can be cascaded to provide its frequency response. This procedure, as well as techniques to extract the transverse wakefields, are at present under investigation.

\section{ACKNOWLEDGEMENTS}

The author is indebted to I. Wilson and W. Wuensch for their support in the course of this study and would like to thank E. Jensen for stimulating and enlightening discussions.

\section{REFERENCES}

[1] Los Alamos Accelerator Code Group, "Poisson / Superfish Reference Manual”, Los Alamos National Laboratory, LAUR-87-126, 1987.

[2] U. Lauströer, U. van Rienen and T. Weiland, "URMEL and URMEL-T User Guide", Deutsches ElektronenSynchrotron DESY, DESY M-87-03, 1987.

[3] U. van Rienen, "Higher Order Mode Analysis of Tapered Disc-Loaded Waveguides Using the Mode Matching Technique", Part. Accel., vol. 41, pp. 171-201, 1993.

[4] M. Dohlus, et al., "Mode Computation in Long Tapered Multi-Cell Linear Accelerator Structures Using the GSM Method”, IEEE MTT-S Int. Microwave Symp. Dig., pp. 1523-1526, Denver, 1997.

[5] V. Dolgashev, et al., "Scattering Matrix Analysis of the NLC Accelerating Structure", Proc. PAC 99, pp. 28222824, New York, 1999.

[6] CLIC Study Team, "A $3 \mathrm{TeV} \mathrm{e}^{+} \mathrm{e}^{-}$Linear Collider Based on CLIC Technology”, CERN 2000-008, CERN, 2000.

[7] Ansoft Corporation, "ANSOFT HFSS User Guide Manual”, 1999.

[8] M. Dehler, et al., "CLIC Waveguide Damped Accelerating Structure Studies”, Proc. LINAC 96, pp. 734-736, Geneva, 1996.

[9] I. Wilson and W. Wuensch, "Revised Parameters for the CLIC Main Linac Accelerating Structure”, CLIC Note 120, CERN, 1990.

[10] I. Wilson and W. Wuensch, "The CLIC Main Linac Accelerating Structure”, Proc. LINAC 2000, pp. 419-421, Monterey, 2000. 